\title{
LA CONSTRUCCIÓN A URMA + SUBJUNTIVO/INFINITIVO COMO MARCA DE FUTURO PLANEADO
}

\author{
MiHAELA TOPOR \\ Escola Universitària Relacions Laborals \\ topormihaela@gmail.com
}

\section{RESUMEN}

El objeto de estudio de este artículo se inscribe dentro de la lingüística descriptiva, ya que nos proponemos demostrar que la perífrasis verbal del rumano formada por el verbo a urma 'seguir' y un verbo en subjuntivo o infinitivo (en adelante, a urma + SUBJ/INF) funciona como una marca de futuro planeado. Para demostrar que la construcción encierra el valor semántico mencionado nos basamos, por un lado, en estudios acerca de cómo se gramaticalizan las marcas temporales, aspectuales y modales (TAM) en idiomas de distintas tipologías y, por otro, en el análisis de datos empíricos de corpus con respecto a las restricciones accionales y temporales de a urma, así como de otros elementos contextuales que indican que el evento expresado por el subjuntivo o el infinitivo está planificado.

PALABRAS CLAVE: rumano; gramaticalización; perífrasis verbales; A URMA + SUBJUNTIVO/INFINITIVO; temporalidad; futuro planeado.

\section{THE A URMA + SUBJUNTIVO/INFINITIVO CONSTRUCTION AS A MARK OF SCHEDULED FUTURE}

\section{ABSTRACT}

We can classify this article within the area of descriptive linguistics, since we propose to demonstrate that the Romanian verbal periphrasis formed with the verb a urma 'to continue' and a subjunctive or infinitive form (from now on, a urma + SUBJ/INF) is a grammaticalized expression for the scheduled future meaning. To demonstrate that the construction encloses the mentioned semantic value we base our analysis on, on the one hand, studies on how temporal, aspectual and modal categories (TAM) grammaticalize in typologically different languages and on the other hand, the analysis of empirical information from corpus regarding the temporal and actional restrictions of a urma as well as of other contextual elements that indicate that the event expressed by the subjunctive or the infinitive is scheduled.

KEY WORDS: Romanian; grammaticalization; verbal periphrases; A URMA + SUBJUNTIVO/INFINITIVO; tense; scheduled future.

\section{INTRODUCCIÓN}

La idea de abordar un estudio de la construcción a urma + SUBJ/INF surge como resultado de varios trabajos previos cuyo objeto de investigación ha sido el análisis contrastivo de las perífrasis verbales del español y del rumano. ${ }^{1}$ Mediante dicho análisis se ha puesto de relieve que el equivalente en rumano de la perífrasis ir a + INF usada con valor temporal de futuro es la construcción formada con el verbo a urma, que en el uso léxico corresponde al verbo seguir del español, y una forma verbal personal

${ }^{1}$ Por perífrasis verbal entendemos la construcción indisoluble formada por dos verbos, en que el primero, como consecuencia de un proceso de gramaticalización, contiene información semántica -de tipo aspectual, modal o temporal- e información gramatical relacionada con el número, la persona, el tiempo y el modo, y el segundo verbo expresa la acción verbal.

Data de recepció: 05/IX/2013 
de subjuntivo o bien una forma no personal de infinitivo. ${ }^{2}$ Dado que en nuestro corpus hay combinaciones de a urma tanto con el infinitivo que con el subjuntivo -concretamente, hay 105 ejemplos con el infinitivo y 95 con el subjuntivo-, nos referiremos a la construccion con a urma + SUBJ/INF. ${ }^{3}$

Ahora bien, a diferencia de lo que ocurre en español, se ha observado que la construcción del rumano, además del valor de futuro, contiene un valor de planificación. En otras palabras, el evento expresado por el infinitivo o el subjuntivo tendrá lugar porque ha sido planificado o preestablecido por un agente, una entidad, etc. Para este tipo de futuro en las gramáticas inglesas se usa el término scheduled future (Raden y Dirven 1984, Comrie 1985) y también profuturo, si nos referimos al español (Marcos Marín et al. 1998).

En lo que se refiere al marco teórico, este estudio se inscribe en el marco teórico de la gramaticalización, proceso mediante el cual una pieza o estructura léxica se convierte paulatinamente en una pieza gramatical, y que se manifiesta en distintos planos: en el plano semántico, mediante la pérdida semántica o desemantización; en el plano pragmático mediante un proceso de extensión, es decir, de obtención de nuevos significados gramaticales cuando la construcción se extiende a otros contextos; en el plano morfosintáctico se manifiesta mediante un proceso de descategorización, que consiste en la pérdida de propiedades morfosintácticas, como la capacidad de selección de argumentos; por último, en el plano fonético se produce una erosión o reducción fonética (Heine y Kuteva 2007). ${ }^{4}$

${ }^{2}$ En rumano ha tenido lugar un proceso de sustitución del infinitivo, una de las formas no personales típicas de las construcciones perifrásticas de los idiomas románicos, por la forma personal de subjuntivo. No obstante, dicho proceso se ha manifestado de forma distinta en la combinación con un verbo u otro. En algunos casos se ha alargado hasta el siglo XIX o incluso hasta más tarde; así, hay estructuras con verbos aspectuales que han mantenido el infinitivo hasta hoy día, como es el caso de a începe, a porni, a prinde, todos con el significado de 'empezar $\mathrm{a}$ ', y hay verbos modales, como a vrea 'querer', a trebui 'deber', que desde los primeros textos escritos aparecen tanto con el subjuntivo como con el infinitivo y, en estos casos, el proceso de sustitucion ha sido muy rápido (Francu 2000).

${ }^{3}$ Las diferencias señaladas al respecto del uso de las dos formas verbales es que la forma de subjuntivo es utilizada más bien en la lengua hablada, mientras que "en el lenguaje escrito, sobre todo en el estilo culto (literario, científico, jurídico) el infinitivo [...] es más frecuente" (Manea et al. 2005: 393).

${ }^{4}$ En el caso de a urma, con la excepción de la erosión, que no es imprescindible para que se pueda hablar de un proceso de gramaticalización, se produce tanto el fenómeno de la desemantización, tal como se muestra en la sección 3.2, como el de descategorización, es decir, el segundo verbo no puede ser sustituido mediante categorías funcionalmente equivalentes, como un pronombre o un sintagma nominal. Por ejemplo, en el caso de i), el verbo a deveni no se puede sustituir por el SN devenire (ii).

i) Actul normativ urmeaza a deveni operant incepind cu 1 iulie 1999. (Evenimentul zilei)

'Está previsto que el acta normativa se haga efectiva a partir del 1 de julio de 1999'

ii) Actul normativ urmeaza *devenire incepind cu 1 iulie 1999.

'El acta normativa sigue *efectividad a partir del 1 de julio de 1999'

En lo que se refiere al proceso de extensión, consideramos que dicho proceso queda reflejado en que el verbo a urma es un verbo polisémico. 
El artículo se estructura de la manera siguiente: en el apartado 2 empezamos por definir el valor de futuro planeado; a continuación, mostramos cómo se expresa en algunos de los idiomas que lo contienen. El apartado 3 contiene, en primer lugar, la información relativa a las acepciones del verbo a urma en el uso léxico, significativa a la hora de explicar la transformación de éste en un elemento gramatical con valor de futuro planeado (3.1). En el segundo subapartado nos dedicamos a describir los valores semánticos que se le han atribuido a a urma + SUBJ/INF en tanto que perífrasis verbal (3.2). En el subapartado final justificamos que el valor con el que se usa hoy día la construcción es exclusivamente el valor de futuro planeado (3.3). Para ello nos basamos en el análisis de ejemplos de corpus con respecto a las restricciones accionales y temporales así como de elementos contextuales que refuerzan la idea de planificación. El apartado 4 contiene las conclusiones a las que llegamos a partir de este estudio, y va seguido de las referencias bibliográficas empleadas.

\section{2. ¿QUÉ ES EL FUTURO PLANEADO?}

Tal como se las define en Bybee et al. (1994: 249), las marcas de futuro planeado son morfemas gramaticales "which refer either to events which are expected to occur in the near future, or to those which have been pre-aranged, which are sometimes referred to as «scheduled futures»". ${ }^{5}$

En Fleischman (1982: 145-146), el término es utilizado en referencia a la construcción devoir + INF del francés, cuya equivalencia semántica con a urma del rumano señalan Iordache y Scurtu (1998): “The basic meaning of the devoir construction is most accurately described as one of prospection, with the added sense of something decided or agreed upon in advance, i.e. scheduled, and scheduled to take place in the foreseeable future".

Tal como se subraya en Bybee et al. (1994), los gramemas de futuro planeado son relativamente poco frecuentes. Concretamente, de un muestreo de setenta y seis idiomas consultados, solo en cuatro se ha detectado la existencia de dicho gramema. En lo que respecta a los idiomas románicos, hasta donde sabemos, dicho valor no está expresado por recursos gramaticalizados como las perífrasis verbales. ${ }^{6}$ Por tanto, consideramos de extrema importancia poder señalar la existencia, en el grupo de dicha familia lingüística, de una estructura que ha gramaticalizado este tipo de significado.

En lo que se refiere a cómo se originan las marcas de futuro, en el trabajo de Bybee et al. (1994), enfocado a describir patrones de gramaticalización de categorías aspectuales, temporales y modales, se menciona que hay una serie limitada de estructuras a partir de las cuales se pueden formar. Así, el futuro se puede formar a

${ }^{5}$ En la categoría de morfemas gramaticales los autores incluyen tanto afijos, auxiliares, como estructuras más complejas, como podrían ser las perífrasis verbales.

${ }^{6}$ Las referencias bibliográficas consultadas acerca de las perífrasis verbales de los distintos idiomas románicos son: Fernández de Castro (1999), García Fernández (2006, 2012) y Olbertz (1998) para el español; Squartini (1998) para el italiano; Lamiroy (1991, 2004, 2011) para el francés y Laca y Gavarró (2002) para el catalán. El hecho de que hayamos revisado la categoría de las perífrasis verbales y no otra clase de elementos gramaticales con la finalidad de localizar ejemplos similares a los de a urma + SUBJ/INF, se debe a que en los idiomas románicos es normalmente a través de los auxiliares perifrásticos que se expresan típicamente las categorías de tipo TAM. 
partir de estructuras que expresan movimiento, obligación, deseo, habilidad y, finalmente, a partir de adverbios temporales. En referencia al futuro formado a partir de estas estructuras, los autores emplean el nombre primary futures. En segundo lugar, el futuro también se puede crear a partir de formas cuyo principal uso es el de expresar el presente, o el aspecto perfectivo o imperfectivo. Este segundo tipo de futuros se llaman aspectual futures. En el muestreo de idiomas analizados por Bybee et al. (1994), solo el inuit dispone de marcas gramaticales, como el sufijo sussaa, cuya función única es expresar el futuro planeado. Las otras tres marcas de futuro planeado documentadas son del segundo tipo, ya que proceden de formas cuya función principal es la de expresar aspecto. Por ejemplo, tanto en latín como en griego, la función de futuro planeado recae, al igual que en inglés, sobre una forma verbal de presente y un modificador temporal. Ateniéndonos a esta clasificación, a urma + SUBJ/INF se comportaría como un futuro del primer tipo en tanto que se desarrolla a partir de un verbo de movimiento.

Se consideran casos de futuro planeado las estructuras mediante las cuales se expresan horarios de tren, fechas en que está previsto que haya exámenes, eventos deportivos, etc. (Günter Raden y René Dirven 1984). En inglés hay tres formas de expresar el futuro planeado: mediante el presente de indicativo (1), el progresivo (2) y la construcción formada con el verbo to be y una forma de infinitivo (3):

(1) We fly to Irkutsk on Wednesday.

'Miercuri zburăm la Irkutsk'

‘El miércoles volamos a Irkutsk'

(2) We're flying to Irkutsk on Wednesday.

'Miercuri zburăm la Irkutsk'

‘El miércoles volamos a Irkutsk'

(3) Maria is to sing in Aida tomorrow night.

'Maria va cânta în Aida mâine seară'

'María cantará en Aida mañana por la noche'

Las gramáticas coinciden en mencionar que, normalmente, el momento en que se produce el evento queda especificado mediante un sintagma con valor temporal. En los ejemplos de (1-3), dichos sintagmas serían on Wednesday (1-2) y tomorrow night (3).

Teniendo en cuenta los ejemplos de (1-3), podría interpretarse que el responsable del significado que estudiamos aquí es el sintagma con valor temporal. No obstante, Comrie (1985) advierte de que el significado de futuro planeado no viene dado exclusivamente por el modificador temporal. Un caso como el de (4), a pesar de tener la misma estructura sintáctica que el ejemplo de (1), no puede interpretarse con el valor de futuro planeado porque los eventos meteorológicos -llover- no pueden ser planificados.

(4) It rains tomorrow.

'Mâine plouă'

‘Mañana llueve' 
Ahora bien, el autor advierte igualmente de que una forma verbal de presente que no vaya acompañada de un modificador temporal adecuado no puede anclarse en el futuro. Por consiguiente, lo que asegura la lectura de futuro planeado es la combinación entre los dos elementos, el verbo en presente y el modificador temporal.

Nuestra hipótesis es que hay indicios de que en rumano, aparte de una construcción como la de (1), la marca de futuro planeado está gramaticalizada en la estructura a urma + SUBJ/INF, que se podría parafrasear como 'está previsto/planificado que se realice la acción expresada por el segundo verbo'. Uno de los indicios que sustentan nuestra hipótesis es que, como veremos en la siguiente sección, la construcción que aquí nos ocupa presenta las condiciones que favorecen la evolución hacia un significado de futuro, concretamente, el significado de movimiento espacial del que ha derivado el de movimiento en el tiempo. Por otro lado, el hecho de que a urma exprese algo más que acción venidera se explica porque el rumano ya dispone de tres formas para expresar el futuro: (i) el auxiliar (AUX) a vrea 'querer' (5); (ii) la forma de infinitivo, con el auxiliar a avea 'tener' y el subjuntivo presente; (iii) la forma invariable $o$ y el subjuntivo presente, estas dos últimas consideradas formas coloquiales (Avram 1986):

(5) Ea [va scrie/ are să scrie/ o să scrie] o poezie.

ella AUX. escribir/AUX. que escriba/AUX. que escriba un poema

‘Ella escribirá un poema'

\section{DESCRIPCIÓN DE LA CONSTRUCCIÓN A URMA + SUBJ/INF}

Tal como hemos adelantado, en este apartado procedemos, en primer lugar, a la descripción de los valores semánticos que presenta el verbo a urma cuando se usa como verbo principal o predicativo, ya que algunos de los usos léxicos son precisamente aquellos a partir de los que se pueden originar valores semánticos de tipo TAM (apartado 3.1). En segundo lugar, presentamos los significados que se le han atribuido a a urma en calidad de auxiliar perifrástico en la bibliografía sobre las perífrasis verbales del rumano (apartado 3.2). Finalmente, partiendo de información relacionada con las restricciones accionales del segundo verbo, de los tiempos verbales en los que se puede conjugar a urma como auxiliar, así como de otros elementos contextuales, nos decantamos por atribuirle a a urma + SUB/INF un valor de futuro planeado. Para extraer esta información hemos recurrido a un muestreo de ejemplos de corpus formado por 200 oraciones que contienen la estructura a urma + SUB/INF extraídas del periódico Evenimentul zilei (EVE). ${ }^{7}$

\subsection{A urma como verbo léxico}

Tal como se observa a partir de las definiciones ofrecidas en el Dicționarul explicativ al limbii române (DEX), que citamos a continuación, el verbo a urma puede tener significados relacionados con el movimiento y la temporalidad. El significado de

7 El corpus tiene 50.000.000 de palabras. No obstante, presenta la limitación de que no está lematizado ni anotado morfológicamente, con la consecuencia de que la única manera de obtener ejemplos de una determinada PV ha sido haciendo búsquedas por forma y no por lema. 
movimiento o desplazamiento se manifiesta en las acepciones 1-3: 1. "A merge în urma cuiva" 'ir detrás de alguien' (6); 2. "A merge înainte pe un anumit traseu" 'seguir adelante por una determinada ruta' (7); 3. "A frecventa o anumită formă de învățământ" 'frecuentar una forma de enseñanza' (8):

(6) Urmează-mă!

sigue me

‘Sígueme!'

(7) Calatorii au urmat acelasi drum timp de doua ore. ${ }^{8}$

viajeros_los han seguido mismo camino tiempo de dos horas

'Los viajeros han seguido el mismo camino durante dos horas'

(8) A urmat facultatea de Drept.

ha seguido carrera de derecho

'Ha hecho la carrera de Derecho'

También puede usarse con un significado temporal, como en la acepción 4. "A veni, în timp, după cineva sau ceva" 'seguir, cronológicamente, a algo o a alguien' (9), y un significado aspectual de continuación, como en la acepción 5. "A duce mai departe un lucru început” ‘seguir haciendo una cosa ya empezada' (10):

(9) După fericire a urmat coşmarul.

después felicidad ha seguido pesadilla_la

'Después de la felicidad llegó la pesadilla'

(10) Fiecare şi -a urmat treaba.

cada_uno PRON ha seguido trabajo

'Cada uno ha seguido con sus tareas'

La acepción 6. recoge un uso intransitivo de a urma: "A avea loc, a se produce, a se îndeplini (după...)" tener lugar, producirse, cumplirse (después...):

(11) Ceea ce a urmat a mulțumit pe toată lumea.

lo que ha seguido ha contentado a todo mundo_el

'Lo que siguió a continuación contentó a todo el mundo'

Por último, en la acepción 7. se le atribuye el valor de auxiliar y, como tal, es considerado sinónimo de los auxiliares a trebui y a fi, ambos con valor de necesidad $\mathrm{y} / \mathrm{u}$ obligación "(Cu valoare de auxiliar) a trebui, a fi nevoie" (con valor de auxiliar) deber, ser necesario (12):

8 Usamos el signo "_" para mostrar que una palabra de una lengua se traduce en la otra por más de una palabra; en el ejemplo (7), viajeros_los corresponden a una sola palabra del rumano, calatorii, ya que en esta lengua el artículo definido es enclítico. 
(12) Acelasi raport arata ca nici o persoana fizica nu urmeaza sa plateasca datoriile $\mathrm{cu}$ propriile bunuri imobile (ziarulevenimentul.ro/.../birurile-neplatite-la-fisc-..)

'El mismo informe muestra que ninguna persona física tiene que pagar las deudas con sus propios bienes inmuebles'

\subsection{A urma + SUBJ/INF como auxiliar perifrástico}

Tal como se ha adelantado, el verbo a urma, además de usarse como verbo léxico, tiene valor de auxiliar perifrástico en la combinación con el infinitivo o el subjuntivo. En este tipo de estructuras se le han asignado distintos valores semánticos. En primer lugar, autores como Hazy (1965) afirman que a urma es un verbo que puede expresar el aspecto durativo:

(13) Ziua a doua de crăciun urmau să o petreacă toți la Gogu. (Hazy 1965: 295).

día segundo de Navidad seguían que la pasen todos a Gogu

'El segundo día de Navidad iban a pasarlo todos en casa de Gogu'

No obstante, en nuestra opinión dicho valor no queda reflejado, puesto que, como se observa en la traducción, el evento pasar ni tan solo ha empezado y, por tanto, tampoco puede ser concebido como durativo.

En segundo lugar, autores como Guțu-Romalo (1961), Trandafir (1973), Iordan y Robu (1978) y Manea et al. (2005) han defendido que este complejo verbal expresa la continuación o la fase intermedia del desarrollo de un evento:

(14) Nu răspunse nimic Anei, ci urmă să privească cu jind la ceata flăcăilor. (Trandafir 1973: 115)

no respondió nada Ana_a sino siguió que mire con anhelo grupo_el mozos

'No le contestó nada a Ana, sino que continuó/siguió mirando con anhelo al grupo de mozos'

En el caso de (14), el valor de continuación queda reflejado en la posibilidad de parafrasear la estructura a urma + SUBJ mediante una construcción perifrástica que expresa el mismo valor, y que es a continua + SUBJ/INF 'continuar + GER'.

En tercer lugar, autores como Iordache y Scurtu (1998) y Dragomir (2003) observan en a urma + SUBJ valores temporales de inminencialidad (15) y futuro indeterminado (16), etiqueta atribuida probablemente porque el conjunto verbal no indica por sí solo el momento futuro en que se va a producir el evento:

(15) Urma să meargă la părinţii lui.

seguía que vaya a padres sus

'Iba a ir a casa de sus padres'

(16) Urma s- o revăd.

seguía que la vuelva_a_ver

'Iba a volver a verla'

En Iordan y Robu (1978: 593) se menciona igualmente un uso con valor temporal de a urma, pero sin más aclaraciones: 
(17) Dînșii urmează a_răspunde mîine.

ellos siguen responder mañana

'Ellos van a dar una respuesta mañana'

En trabajos más recientes sobre la construcción que nos ocupa, como el de Comes (2003), se defiende igualmente el valor temporal de a urma cuando es usado en construcciones perifrásticas. Para ello, la autora se sirve de tres argumentos. En primer lugar, todo el conjunto verbal puede ser parafraseado por una forma de futuro, como mostramos en (18) a partir del ejemplo de (17):

(18) Dînșii vor_răspunde mîine.

ellos contestarán mañana

'Ellos contestarán mañana'

En segundo lugar, el verbo a urma se puede conjugar en distintos modos y tiempos verbales, menos en futuro, dado que el significado que expresa ya es un significado de futuro. En tercer lugar, la estructura es compatible con expresiones que indican valor temporal de posterioridad.

Ahora bien, en referencia al valor presentado en (14), Comes señala que los casos en que a urma es usado con valor de continuación son muy escasos, ya que en su corpus, formado por 250 páginas de textos literarios y periodísticos, la autora detecta pocas ocurrencias en que a urma es usado con significado de continuación. Concretamente, localiza 3 usos en que a urma es empleado como verbo predicativo con significado de movimiento en el espacio, como en la acepción 1.; 3 usos con significado temporal, como en la acepción 4; 9 usos con el significado léxico de continuación mostrado en la acepción 5, y sólo 1 uso como verbo gramaticalizado que expresa aspecto continuo. Con respecto a la capacidad de a urma de expresar aspecto continuo, la conclusión a la que llega la autora es que las ocurrencias de este tipo han caído en desuso y que se ha extendido el valor temporal de futuro.

En nuestra opinión, este tipo de evolución no debería extrañar si el idioma ya dispone de recursos para expresar un determinado significado, en este caso el de continuación, que en rumano se realiza mediante la perífrasis verbal a continua + SUBJ 'continuar + GERUNDIO'. Por otro lado, tampoco es de extrañar la evolución de a urma hacia el significado temporal, ya que es ampliamente conocido que las construcciones que expresan movimiento espacial tienden a dar lugar a gramemas con valor de desplazamiento temporal. Es lo que ha ocurrido con el verbo go en inglés, que ha dado lugar a la construcción progresiva, o con los verbos aller e ir del francés y español, respectivamente, que se han convertido en auxiliares perifrásticos con valores temporales de futuro e inminencialidad (cf. Larreya 2005 para el francés y Bravo 2008 para el español).

\subsection{A urma + SUBJ/INF como marca de futuro planeado}

Para sostener que en el caso de a urma + SUBJ/INF se ha de hablar de futuro planeado y no solamente de futuro, nos basamos en tres tipos de datos: los referentes a las restricciones accionales y temporales; otros elementos contextuales, como los que indican el momento en que está prevista la realización del evento designado por el V2, 
que puede aparecer explícito o no; los que indican el agente o la entidad responsable de la planificación de dicho evento.

En lo que atañe al tipo de clase accional que puede expresar el verbo en infinitivo o subjuntivo, una consecuencia de la especialización semántica del auxiliar a urma es que no cualquier tipo de verbo puede actuar a su lado como verbo principal. El uso de a urma como futuro planeado impide que los predicados que expresen eventos que no puedan ser planificados actúen como verbos principales. En primer lugar, los predicados estativos permanentes que expresan propiedades inalienables no pueden aparecer al lado de a urma por el simple hecho de que no se pueden planificar las características físicas de una persona:

(19)*Copilul urmează să fie blond.

niño_el sigue quesea rubio

'Está previsto que el niño sea rubio'

En cambio, sí hay compatibilidad entre a urma y los predicados estativos cuando estos designan propiedades sobre las cuales puede intervenir el agente humano y que consideramos no permanentes. Eso ocurre en el caso de objetos -como casas, escuelas, carreteras- con respecto a cuyas dimensiones deciden los agentes humanos:

(20) România urmează să aibă 1800 de Km de autostradă până în 2013.

Rumanía sigue que tenga 1800 de Km de autovía hasta en 2013

'Está establecido/planificado que Rumanía tenga 1800 Km de autovía hasta el 2013'

No pueden ser verbos principales de a urma aquellos predicados que contengan el semema /-control/, como en (21) :

(21) *Urmează să cazi!

Sigue que caigas

'Está establecido/planificado que te caigas'

o los sememas /-control/ y / +experiencia subjetiva/ que los inhabilitan para expresar futuro planeado:

(22) Dacă îţi dai_jos tricoul *urmează să -ţi fie frig.

Si te quitas camiseta_la sigue que te sea frío

'Si te quitas la camiseta, está establecido/planificado que tengas frío'

Tanto en (21) como en (22), el auxiliar que se debería emplear es el auxiliar a avea y cuyo equivalente en español es ir $a$, capacitado para ser usado con acciones espontáneas (23) o que ocurren como consecuencia de otras acciones (24):

(23) Ai să cazi!

tienes que caigas

'Te vas a caer' 
(24) Dacă îți dai_jos tricoul are să -ţi fie frig.

Si te quitas camiseta_la tienes que te sea frío

'Si te quitas la camiseta vas a tener frío'

En lo concerniente a las restricciones temporales, los datos proporcionados por el muestreo de ejemplos del corpus analizado corroboran los resultados de Comes (2003). En primer lugar, tal como se observa en la tabla 1, que contiene la información relativa a los tiempos y modos verbales en los que se conjuga a urma, el auxiliar muestra preferencia por el presente de indicativo, ya que en más del $75 \%$ de los ejemplos se conjuga en dicho tiempo verbal. Le sigue el imperfecto de indicativo, con 21 ocurrencias, en cuyo caso se obtiene el significado de futuro en el pasado, que en español puede ser expresado con la perífrasis ir a $+I N F$ :

(25) Gindul ca urma sa ne casatorim imi dadea mai_mult spor (EVE).

idea_la que seguía que nos casemos me daba más energía

'La idea de que nos íbamos a casar me daba más energía'

Tal como se puede apreciar, en nuestro corpus solo hay un ejemplo en que a urma es empleado en futuro de indicativo, seguramente debido a la redundancia de significado a la que alude Comes. Asimismo, nuestro corpus confirma la incompatibilidad de a urma con las formas de aspecto perfectivo -pretérito perfecto, pretérito indefinido o pluscuamperfecto- que anularían la interpretación del evento expresado por el subjuntivo o el infinitivo como un evento futuro.

\begin{tabular}{|l|l|}
\hline Presente de indicativo & 159 \\
\hline Imperfecto de indicativo & 21 \\
\hline Futuro de indicativo & 1 \\
\hline Condicional Presente & 18 \\
\hline
\end{tabular}

TABLA 1. Modos y tiempos verbales en los que se conjuga a urma en tanto que auxliar.

El tercer argumento que aducimos a favor de la lectura de futuro planeado es que la perífrasis suele ir acompañada de expresiones que indican el momento exacto en que ha de tener lugar el evento. Concretamente, el momento en que está planificada la realización del evento expresado por el V2, está expresado en 43 de los 200 ejemplos de nuestro corpus mediante adverbios (26), sintagmas preposicionales (27) o subordinadas adverbiales (28.) Esta observación y el hecho de que en nuestro corpus tampoco hayamos detectado usos continuativos de a urma, nos confirma que la construcción ha perdido los valores de continuación y ha extendido el valor temporal.

(26) Sindicatele urmeaza a prezenta guvernului abia astazi_decizia oficiala in legatura cu greva generala (EVE).

‘Está previsto que los sindicatos presenten hoy finalmente al gobierno la decisión oficial con respecto a la huelga general'

(27) Textul acestui proiect de lege urmeaza a fi aprobat in cursul acestei saptamini (EVE). 
'Está previsto que el texto de este proyecto de ley sea aprobado a lo largo de esta semana'

(28) Toata marfa sechestrata urmeaza a intra pe piata imediat ce firmele care le distribuie vor achita contravaloarea analizelor (EVE).

'Está previsto que toda la mercancía intervenida se venda tan pronto como las empresas que la distribuyen abonen el importe de los análisis'

Otro elemento a favor de la interpretación de futuro planeado es, en nuestra opinión, el uso de la perífrasis en contextos en los que o se habla de acciones rutinarias o se anuncian eventos deportivos (29), asuntos políticos (30) o actos administrativos (31), que son programados con antelación.

(29) Luni, de la ora 19.00, urmeaza sa aiba loc si prima partida [...], cu reprezentativa Coreii de Sud (EVE).

'El lunes, a partir de las 19.00, está previsto que tenga lugar también el primer partido [...] con el equipo que representa a Corea del Sur'

(30) Refugiatii urmeaza sa revina in Kosovo imediat dupa retragerea completa a fortelor sirbe (EVE).

'Está previsto que los refugiados vuelvan a Kosovo inmediatamente después de que las fuerzas serbias se hayan retirado por completo'

(31) Alte 19 asociatii care nu si-au achitat datoriile urmeaza a fi sanctionate in aceasta saptamina (EVE).

‘Esta semana está previsto que sean sancionadas otras 19 asociaciones que no pagaron sus deudas'

\section{CONCLUSIONES}

En este artículo hemos mostrado que a la construcción del rumano a urma + SUBJ/INF se le ha de atribuir un valor de futuro planeado, un gramema relativamente poco frecuente no solamente en el conjunto de la familia de los idiomas románicos, sino también en familias lingüísticas de otras tipologías.

Para ello nos hemos basado en distintos tipos de restricciones, accionales y temporales, así como en otros elementos contextuales que indican que el evento expresado por el V2 está planificado. En lo referente a las restricciones accionales, hemos señalado la incompatibilidad del auxiliar con predicados que expresan eventos que ocurren espontáneamente o con predicados estativos que expresan propiedades inalienables. En lo que atañe las restricciones temporales, hemos evidenciado la preferencia del auxiliar por los tiempos imperfectivos, el presente y el imperfecto de indicativo, así como la incompatibilidad con los tiempos perfectivos, que anularían la interpretación de futuro del auxiliar. Asimismo, hemos señalado que la presencia de elementos contextuales que indican cuándo está prevista la realización del evento refuerza la idea de planificación.

Los resultados de esta investigación contribuyen, por un lado, al campo de la traducción -restringida o no a la categoría de las perífrasis verbales- en tanto que ofrecemos información acerca de las condiciones de uso de la construcción aquí 
analizada y, por otro, a la ampliación de la información acerca de cómo se gramaticaliza el gramema de futuro planeado en rumano, a saber, a partir de una estructura léxica de movimiento que evoluciona hacia un valor de movimiento temporal.

\section{BIBLIOGRAFÍA}

AVRAM, L. (1986), "A few remarks on futurity in Romanian", Revue roumaine de linguistique, XXXI: 3, 203-207.

BRAVO, A. (2008), La perífrasis ir a +infinitivo en el sistema temporal y aspectual del español, Tesis doctoral, Universidad Complutense de Madrid. [Consulta: 2 septiembre de 2013]. Disponible en: $<$ http://eprints.ucm.es/8074/1/T30424.pdf $>$.

BYBeE, J., PERKINS, R., PAGLIUCA, W. (1994), The evolution of grammar. Tense, aspect and modality in the languages of the world, Chicago/London, The University of Chicago Press.

COMES, E. (2003), "Statutul de semiauxiliar al verbului a urma", Ovidius University Annals of Philology, vol. XIV, 53-62.

COMrIE, B. (1985), Tense, Cambridge, Cambridge University Press.

DEX (1996), Dicționarul explicativ al limbii române, ediția a II-a revăzută și adăugită, Academia Română, Institutul de Lingvistică „Iorgu Iordan”, București, Editura Univers Enciclopedic.

DRAGOMIR, C. (2003), “Le role de l'aspect dans le glissement d'une classe processuelle à une autre: Les operateurs aspectuels de phase en roumain", en Verbalperiphrasen in den (ibero)romanischen) Sprachen, Pusch, C.D. y Wesch A. (eds.), Hamburg, Helmut Buske Verlag, pp. 113-122.

FERNÁNDEZ DE CASTRO, F. (1999), Las perífrasis verbales en el español actual, Madrid, Gredos.

FLeISCHMAN, S. (1982), The Future in Thought and Language: Diachronic Evidence from Romance, Cambridge, Cambridge University Press.

FRANCU, C. (2000), Conjunctivul românesc și raporturile lui cu alte moduri, Iași, Casa editorială Demiurg.

GARCÍA FERNÁNDEZ, L. (dir.). (2006), Diccionario de perífrasis verbales, Madrid, Gredos.

GARCíA FERNÁNDEZ, L. (2012), Las perífrasis verbales en español, Madrid, Castalia.

GAVARRÓ, A. y LACA B. (2002), "Les perífrasis temporals, aspectuals i modals", en Gramàtica del català contemporani, Solà, J. et al. (dir.), Barcelona, Empúries, pp. 2663-2726.

GUTU-RomalO, V. (1961), “Semiauxiliare de aspect?”, Limba romană, X: 1, 3-15.

HAZY, S. (1965), “Predicate verbale compuse”, Cercetări de lingvistică, X: 2, 289-297.

HeIne, B. y KuteVA T. (2007), The Genesis of Grammar, Oxford, Oxford University Press.

IORDACHE, R. y SCURTU G. (1998), “Autour des formes periphrastiques verbales du français et de leur équivalence en roumain", en Atti del XXI Congresso Internazionale di Linguistica e Filologia Romanza, Tubingen, Max Niemeyer, pp. 483495.

IORDAN, I. y ROBU, V. (1978), Limba română contemporană, Bucureşti, Editura Didactică şi Pedagogică. 
LAMIROY, B. (1991), "Perífrasis verbales", en Léxico y gramática del español: estructuras verbales de espacio y de tiempo, Barcelona, Anthropos, pp. 83-109.

LAMIROY, B. (2004), “La teoría de la gramaticalización y sus aplicaciones en las lenguas románicas", Estudios de lingüística, 18, 245-266.

LAMIROY, B. (2011), “Degrés de grammaticalisation à travers les langues de même famille", Mémoires de la Société de linguistique de Paris. Nouvelle Série, 19, 167-192.

LARREYA, P. (2005), "Sur les emplois de la périphrase ALLER + INFINITIF", en Les Périphrases Verbales, Bat-Zeev Shyldkrot, H. y Le Querler, N. (eds.), Amsterdam, John Benjamins, pp. 337-360.

MANEA et al. (2005), "Verbul”, en Gramatica limbii romane, Bucureşti, Editura Academiei Romane, pp. 323-584.

Marcos Marín, F., SATORRe GraU, F.J., Viejo SÁNCHEZ, Mª́L. (1998), Gramática española, Madrid, Síntesis.

OlBeRTZ, H. (1998), Verbal periphrases in a Functionnal Grammar of Spanish, Berlín/Nueva York, Mouton de Gruyter.

RADEN, G. y DiRVEN R. (1984), Cognitive English Grammar, Amsterdam/Philadelphia, John Benjamins.

SQUARTINI, M. (1998), Verbal Periphrases in Romance. Aspect, Actionality, and Grammaticalization. Berlin/New York, Mouton de Gruyter.

TRANDAFIR, Gh. D. (1973), Categoriile gramaticale ale verbului în româna contemporană, Craiova, Casa corpului didactic al județului Dolj. 